\title{
Principles of Diffusion Kurtosis Imaging and its Role in Early Diagnosis of Neurodegenerative Disorders
}

Authors: Anas Arab ${ }^{1}$, Anna Wojna-Pelczar ${ }^{2}$, Amit Khairnar $^{3 *}$, Nikoletta Szabó ${ }^{2,4}$, Jana RudaKucerova $^{1}$

\section{Author's Affiliations:}

${ }^{1}$ Department of Pharmacology, Faculty of Medicine, Masaryk University, Brno, Czech Republic

${ }^{2}$ Research group Multimodal and Functional Neuroimaging, CEITEC - Central European Institute of Technology, Masaryk University, Brno, Czech Republic

${ }^{3}$ Applied Neuroscience Research Group, CEITEC - Central European Institute of Technology, Masaryk University, Brno, Czech Republic

${ }^{4}$ Department of Neurology, Faculty of Medicine, Albert Szent-Györgyi Clinical Center, University of Szeged, Szeged, Hungary

\section{*Correspondence:}

Amit Khairnar, Ph.D.

Applied Neuroscience Research Group, CEITEC - Central European Institute of Technology, Masaryk University

Kamenice 5, 62500 Brno, Czech Republic

E-mail: amitkhairnar520@gmail.com

Phone: +420 549494 238, Fax: N/A

Keywords: Diffusion kurtosis imaging, neuroimaging, neurodegenerative disorders, Parkinson's disease, Alzheimer's disease, Huntington's disease. 


\section{Abbreviations:}

AK: Axial kurtosis

AlzD: Alzheimer's disease

D: Diffusion coefficient

AD: Axial diffusivity

MD: Mean diffusivity

RD: Radial diffusivity

DWI: Diffusion weighted imaging

DTI: Diffusion tensor imaging

CNS: central nervous system

FA: Fractional anisotropy

GM: Gray matter

HD: Huntington disease

MK: Mean kurtosis

MCI: Mild cognitive impairment

PD: Parkinson's disease

RK: Radial kurtosis

ROI: Region of interest

SN: Substantia nigra

WM: White matter 


\section{Introduction}

The diagnostic use of neuroimaging has increased in the past twenty years. Different imaging techniques are widely available such as computed tomography, positron emission tomography and magnetic resonance imaging (MRI)(1). Neuroimaging can evaluate the early development, ageing and pathological stages of the central nervous system (CNS) and monitor changes during and after treatment (2-4). MRI became an important approach in neuroscience research as well as clinical applications, due to many advantages such as wide spectrum of MRI methods used with different out comes such as strucural and diffusional methods (5), and the ability to visualize non-invasively anatomical structures of the brain or their functional state(6). Since the brain consists of approximately $70 \%$ of water, motion of water throughout the tissues provides an important insight into brain microstructure. The characteristics of diffusion are often associated with the structural organization of tissues. Therefore, one rapidly developing field of MRI based on the water diffusion detection is diffusion weighted imaging (DWI). This technique was introduced fifty years ago when diffusional changes were reported for the first time (7). DWI is a unique MRI technique which maps water motion at microstructural level and it is able to differentiate between normal and abnormal water diffusion in biological tissues (8). DWI considers water motion free in any direction with no hindrance, i.e. isotropic diffusion. DWI is widely used in clinical fields, in diagnosis of acute ischemic stroke, traumatic brain injury and inflammation $(9,10)$. However, all biological systems are complex structures with a variety of barriers restricting the water diffusion and leading to directional water movement. This process is called anisotropic diffusion (8).

\section{Physical theory of the diffusion weighted imaging}

Diffusion is a mass transport, a random movement of molecules or particles (which do not require bulk motion) $(11,12)$. An approach to diffusion was undertaken by Einstein and Smoluchowski, they proposed a displacement distribution parameter describing the mean squared displacement of the particles in timeframe (13).

$$
<x^{2}>=2 D \Delta
$$

where $\mathrm{D}$ is diffusion coefficient, $\left\langle x^{2}\right\rangle$ is the mean squared displacement during a diffusion time $\Delta$. In three-dimension, the equation has a form:

$$
<r^{2}>=6 D t
$$

where $\left\langle r^{2}\right\rangle$ is the mean squared displacement in 3D. The distribution of squared displacements takes a Gaussian form (see Figure 1). Any MR imaging technique can be designed to be sensitive to diffusion by adding magnetic fields gradients. It is possible, with the usage of 
diffusion-sensitizing gradients in any direction (see Figure 2). Diffusion imaging provides an estimate of D in each voxel from a series of DWI obtained with gradients applied in one direction. Spins that are moving along the direction of the applied gradient diphase and, consequently, decrease of the signal, which can be represented with a mono-exponential signal decay equation:

$$
S=S_{0} e^{-b D}
$$

thus:

$$
\ln \frac{S}{S_{0}}=-b D
$$

where $S$ is the measured signal, $S_{0}$ is the signal measured without diffusion gradient $(b=0)$ and $\mathrm{b}\left(\mathrm{s} / \mathrm{mm}^{2}\right)$ is the measure of diffusion encoding in the diffusion gradients:

$$
b=r^{2} \delta^{2}|G|^{2}\left(\Delta-\frac{\delta}{3}\right)
$$

where $\Upsilon$ is the gyromagnetic ratio, $\delta$ is the duration of the diffusion pulse, $|G|$ is amplitude of the diffusion gradient and $\Delta$ is time interval between two diffusion gradients (Figure 2). Parameter is called a $b$-value, which is a factor that reflects the strength and timing of the gradients used in diffusion-weighted images.

It is known that a tissue is characterized with anisotropic diffusion due to membranes or cell wall. This argument imposes diffusion coefficient to be characterized with the $2^{\text {nd }}$ order diffusion tensor ( 3 x 3 matrix):

$$
\boldsymbol{D}=\left(\begin{array}{lll}
D_{x x} & D_{x y} & D_{x z} \\
D_{y x} & D_{y y} & D_{y z} \\
D_{z x} & D_{z y} & D_{z z}
\end{array}\right)
$$

where $D_{x x}, D_{x y} \ldots$ represents different diffusion rates along given direction. $\mathbf{D}$ is positive definite and symmetric. Diffusion tensor can be represented as a triaxle ellipsoid in the reference frame of the eigenvectors $(11,14)$, which simplified scheme is presented on Figure 3. This model simplifies to isotropic model when diagonal elements are equal $D_{x x}+D_{y y}+D_{z z}=$ $D$ and off-diagonal elements equal zero.

DTI assumes that diffusion can be described by a tensor and was developed to describe anisotropic diffusion (11). Here, minimum six gradient directions and two b-values are needed to calculate DTI map from tensors (tensor has $3^{2}=9$ components and 6 are independent). In this modality the most clinically useful measure obtained from DTI are MD and anisotropy indices characterized by FA or relative anisotropy (RA) (12). MD is represented as:

$$
M D=<D>=\frac{D_{x x}+D_{y y}+D_{z z}}{3}=\frac{\lambda_{x x}+\lambda_{y y}+\lambda_{z z}}{3}
$$


and is linked with tissue integrity (white and grey matter). The most common parameter used is FA, which normalizes the variance by the magnitude of the tensor as a whole:

$$
F A=\sqrt{\frac{3}{2} \frac{\left(D_{x x}-<D>\right)^{2}+\left(D_{y y}-<D>\right)^{2}+\left(D_{z z}-<D>\right)^{2}}{D_{x x}^{2}+D_{y y}^{2}+D_{z z}^{2}}}
$$

FA describes the fraction of the tensor that can be assigned to anisotropic diffusion, thus shows regions where diffusion is most directional (describes integrity of the WM fiber bundles) (11, $15,16)$. Another handy representation is given by principal diffusion directions such as AD and RD, which are defined below:

$$
A D=D_{x x}, R D=\frac{D_{y y}+D_{z z}}{2}
$$

$\mathrm{AD}$ and RD can identify axonal injuries or demyelination (17) An extension of DTI model, called DKI is described. It was introduced in 2005 and since then, many theoretical papers demonstrated its complementary value to DTI $(18,19)$. This model assumes that water diffusion in biological systems has a non-Gaussian behavior. In statistic, kurtosis is a parameter, which describes peakedness of a probability of water distribution (see Figure 1). Higher values of kurtosis assume more complex environment and aberrations to normal diffusion. In DKI model logarithmic signal loss can be described as:

$$
\ln \frac{S}{S_{0}}=-b D_{a p p}+\frac{1}{6} b^{2} D_{a p p}^{2} K_{a p p}
$$

where $\mathrm{K}$ is kurtosis. Kurtosis is related to the $4^{\text {th }}$ order tensor $(3 \times 3 \times 3 \times 3)$.

Kurtosis tensor $(\mathrm{K})$ has complex form and geometrically kurtosis cannot be represented as an ellipsoid.

The higher the kurtosis, the more the water molecule diffusion deviates from Gaussian distribution pointing at more restricted diffusion environment. The most practical parameters are $\mathrm{MK}, \mathrm{AK}$ and $\mathrm{RK}$.

$$
M K=\frac{1}{n} \sum_{i=1}^{n} K_{i}, A K=K_{1}, R K=\frac{K_{2}+K_{3}}{2}
$$

MK is defined as the kurtosis averaged among all directions. Respectively, FA is defined as:

$$
F A_{K}=\sqrt{\frac{3}{2} \frac{\left(K_{1}-\bar{K}\right)^{2}+\left(K_{2}-\bar{K}\right)^{2}+\left(K_{3}-\bar{K}\right)^{2}}{K_{1}^{2}+K_{2}^{2}+K_{3}^{2}}}, \bar{K}=\frac{1}{3} \sum_{i=1}^{3} K_{i}
$$

The DKI method requires data acquisitions with several (minimally three) $b$-values (with bvalue usually larger than $1000 \mathrm{~s} / \mathrm{mm}^{2}$ ) and at least 15 directions of diffusion gradient (18). The 
mono-exponential model DTI is valid only for small b-values (Figure 3). When larger b-values are used (larger than $1000 \mathrm{~s} / \mathrm{mm}^{2}$ ) the method is sensitive to shorter molecular distances showing deviation from DTI mono-exponential model and allows probing regions with larger diffusion restrictions. A simple illustration associated with DTI and DKI models is presented on Figure 4. The main limitation in DTI is the strict dependence of the results (e.g. values of MD or FA) on the used b-values, which can lead to misinterpretation of the results. In DKI, restricted diffusion can be investigated when high b-values are used, even values $800-15000$ $\mathrm{s} / \mathrm{mm}^{2}$ probing $1-2 \mu \mathrm{m}$ molecule distances and diffusion of order of $50-100 \mathrm{~ms}$ (14). Additionally, in some studies data were acquired for many diffusion directions, up to 100 (2), yet, such large number of gradient directions imposes longer measurement as brain images are repeated multiplied by the number of used b-values. Longer scanning has to be taken under consideration especially in terms of motion artefacts caused by subject's movement in the scanner.

\section{Diffusion tensor imaging (DTI)}

The biological organized structure of the CNS including myelinated axons, unmyelinated axons, cellular membrane, presence of proteins and intracellular organelles affect the water diffusion inside the CNS. This factors could divide the water diffusion in CNS into two types, isotropic diffusion in which water diffusion is equal in all directions and is observed in cerebrospinal fluid and grey matter. The second type is anisotropic diffusion, in which water diffusion is mostly unidirectional and is observed in white matter. This technique that accounts for anisotropic directionality is called DTI. It describes the magnitude of the water diffusion in three dimensions and characterizes the directionality of water movement in fibrous tissues showing their architecture (20). DTI considers that the water diffusion in each voxel follows Gaussian distribution $(21,22)$. According to this assumption a biological system is similar to a bucket of water without diffusion hindrance (23). However, brain tissues are much more complex and in truth water diffusion is hindered by cell membrane, cell constituents and it follows non - Gaussian distribution. This is important especially in white matter (WM) where myelinated and unmyelinated axons represent highly fibrous structures rendering the diffusion anisotropic (24). Specifically, water diffusion in WM is relatively unrestricted parallel to the fiber (axonal) orientation while it is highly restricted in the perpendicular direction (24). Gray matter (GM) which consists mainly of neuronal cell bodies where diffusion is more isotropic, so water diffusion unlike WM is unrestricted in all directions (25). However, majority of the studies used fractional anisotropy (FA) as the main DTI metric. FA measures the degree of the 
diffusional anisotropy in the brain tissue such as the extent of differences among the three directions. FA is a derived value and has a range between 0.1 and 1 . The increase of FA values reflects directional coherence or diffusion restriction, which means that the extent of diffusion in one direction. However, the exact biological processes leading to changes in the diffusivity parameter are still unknown. Lower FA might be associated with changes in intrinsic properties of a fiber or changes due to the presence of more than one fiber in a voxel. FA fails to detect changes in the gray matter (GM), because the diffusivity is more isotropic, i.e. water diffuses almost freely in all directions, while FA is more sensitive in directionally oriented structures like in WM $(18,21)$. Thus, validity of the DTI results is limited in GM, while it allows to distinguish between normal and pathological state of the WM. More directionally specific DTI metrics: axial diffusivity (AD), radial diffusivity (RD) and mean diffusivity (MD), which distinguished by direction. AD measures the extent of diffusion occurring in parallel direction with the fiber and it can be altered by deterioration of the axonal integrity (26). RD reflects the extent of diffusion occurring perpendicular to the fiber and therefore RD has an inverse relation with the myelin integrity degeneration (26). MD is the mean of $\mathrm{AD}$ and $\mathrm{RD}$ describing the magnitude of the diffusion.

DTI has been applied in a wide range of clinical and preclinical research studies of CNS and increase the high light on this method for diagnosis of neurodegenerative processes such as multiple sclerosis $(27,28)$, Alzheimer's disease $(29,30)$ and Parkinson's disease $(31)$ or other diseases such as stroke (32) and traumatic brain injury (33).

\section{Diffusion kurtosis imaging (DKI)}

DTI considers the diffusion of water in brain as Gaussian distribution, hence it is unable to detect the diffusion heterogeneity in biological tissues. Therefore, DKI technique was introduced as a mathematical extension of DTI. Kurtosis is described as a dimensionless measure which quantifies the non-Gaussian distribution of water diffusion in a voxel $(18,21)$. Thus, DKI also considers the voxel in the biological system as a bucket of water but with diffusional hindrance arising from multiple tissue compartments, thus it detects restriction of the water diffusion in isotropic as well as anisotropic environment of each voxel according to the degree of restriction such as protein accumulation, while FA is able to detect the restriction of water diffusion only in the anisotropic environment (23). DKI provides not only the diffusion tensor metrics ( $\mathrm{AD}, \mathrm{RD}, \mathrm{MD}$, and $\mathrm{FA}$ ) but also the kurtosis metrics: axial kurtosis (AK), radial kurtosis (RK), and mean kurtosis (MK). The higher the kurtosis value the more restrictions of the normal diffusion inside each voxel wether there is some factors that affects the water 
diffusion even if it is not an anisotropic enviroment, unlike FA which consider the extent of diffusion in one direction which can be seen in the anisotropic environment only. This can be caused by different factors, e.g. pathological protein accumulation or iron deposition while decrease of kurtosis could be a sign of neuronal loss. Thus, DKI is sensitive in detecting pathology in the GM (34) as well as in WM (35). Therefore, the importance of DKI comes from the ability to detect the anisotropic as well as the isotropic diffusion. This supports the possibility that DKI might become a sensitive early stage biomarker for many neurodegenerative disorders.

\section{Preclinical and clinical applications of DKI \\ Parkinson's disease}

Parkinson's disease (PD) is the second most common progressive neurodegenerative disorder characteristic by motor symptoms represented by bradykinesia, resting tremor, muscle rigidity and gait problems. The PD patients also suffer numerous non-motor symptoms including cognitive dysfunction, sleep disorders, psychiatric comorbidities, olfactory and gastrointestinal dysfunctions (36). Furthermore, motor PD symptoms start when 50-70\% of dopaminergic projections from substantia nigra $(\mathrm{SN})$ to striatum are lost, i.e. at a very late stage of the neurodegenerative process. PD neuropathology progression involves multiple processes, including intracytoplasmic abnormal aggregation of protein in neurons called Lewy bodies, which are mainly consist of $\alpha$-synuclein and ubiquitin $(37,38)$. $\alpha$-synuclein is a protein which is found pre-synaptic ally, the accumulation of $\alpha$-synuclein induce the formation of toxic oligomeric protein which can activate the immune system and lead to degeneration of the neurons (39). The etiology of PD remains to be elucidated and many factors such as aging, environmental and genetics contribute the neurodegeneration in PD, diffusion weighted MRI techniques may be able to efficiently track the pathological process.

Many theories were postulated to explain the mechanism behind neuronal loss, such as oxidative stress, rise of iron content, pathological protein accumulation and neuroinflammation $(40,41)$. One theory which describes the origin of idiopathic PD is the Braak's theory. It postulates that PD starts when $\alpha$-synuclein accumulation initiates through olfactory bulbs, dorsal motor nucleus of the glossopharyngeal vagal nerve. Then, at the second stage $\alpha$ synuclein accumulation spreads through the brain regions such as brain stem, medulla oblongata which may develop the non-motor symptoms such as cognitive dysfunction and depression and when the pathology reaches SN the motor symptoms start to appear. (38). In 
the later stages the accumulation spreads to more brain regions such as the striatum and cortex $(39,42)$.

Hitherto the treatment of PD is based on increasing the level of dopamine which does not stop the protein accumulation neither the neurodegeneration process. Thus, the development of reliable early stage diagnosis may help in the development of neuroprotective treatments, which are needed to halt or slow the PD progression when patients might still be eligible for neuroprotective treatment $(43,44)$. DKI seems to be a promising candidate and both clinical and preclinical studies are currently trying to evaluate its relevance as such biomarker.

\section{Clinical studies}

Several DTI studies in PD patients have demonstrated that FA is reduced in SN at both early and late stage of PD $(45,46)$. Furthermore, DTI detected a decrease in FA and increase of diffusivity caused the olfactory dysfunction which might be one of the initial phases of PD (47, 48). However, DTI sensitivity is limited to detect only the anisotropic diffusivity changes that is relevant only in the WM. Therefore, several clinical studies used DKI in PD patients in order to detect the changes in both WM and GM.

Wang et al.(49) were the first to find a significant increase in MK in caudate nucleus, putamen, globus pallidus and $\mathrm{SN}$ in the early to middle stage of PD and suggested that MK reflects the presence PD pathology rather than the stage of disease. However, they admitted that the explanation of MK changes in basal ganglia of PD patients is yet unknown. Unfortunately, AK and RK data were not evaluated in this study. However, the results of AK and RK may provide more information about the mechanisms underlying the DKI changes in PD patients (49). The first to apply DKI on WM of PD patients was Kamagata et al., in two different studies. In the first study MK and FA were found to be decreased in anterior cingulum in PD patients while MK gave the best diagnostic performance. Importantly, the authors claimed that anterior cingulum is the part of brain that shows a pathological alteration at early stage and MK in this region may be useful as a PD diagnostic biomarker (50). Furthermore, the same team used tract-based spatial statistics analysis which identifies the core of the WM bundles and with this method alteration of diffusivity parameters can be detected for evaluation of MK of the whole brain (51), MK was reduced in frontal WM, corpus callosum, parietal, occipital, and right temporal lobe and by analyzing the crossing fibers areas, MK was also reduced in PD patients in posterior corona radiata and superior longitudinal fascicle (52).

The most recent study by Zhang et al (53), assessed DKI in the SN and compared the sensitivity of MK over FA in this region. A significant increase in MK and decrease in FA were found in 
this study (53), they concluded that MK results goes in line with Wang et al. (49) the only study concerning DKI in SN of PD patients. Also FA decrease is consistent with other clinical foundations in the same brain region $(54,55)$. Based on the previous studies MK found to be increased in GM and decreased in WM in different brain region of PD patients. In conclusion, based on the results from clinical studies MK seems to be able to help the clinical diagnosis due to the detection of early changes in PD, especially in the SN. Unfortunately, there is no data out of other DKI matrices such as AK, RK and their diagnostic value is currently unknown.

\section{Preclinical studies}

Just like in case of the clinical studies, there are only a limited number of studies on PD-like animal models reporting DTI and DKI findings. So far, three different studies used pharmacological models of PD-like neurodegeneration, specifically - MPTP (1-methyl-4phenyl-1, 2, 3, 6-tetrahydropyridine) treated mice, and 6-hydroxydopamine (6-OHDA) lesioned rats. DTI results demonstrated a reduction of FA in $\mathrm{SN}$, which goes in line with the DTI clinical studies $(56,57)$. A recent study used marmosets treated with MPTP to study the nigrostriatal pathway. MD was increased in the right and AD in the left side while RD was increased in both sides of the nigrostriatal pathway. Interestingly no significant FA results were found in SN and the nigrostriatal pathway unlike the previous studies (58). However, as repeatedly pointed out, DTI results may not be valid enough in assessment of GM structures.

Khairnar et al. were the first who applied DKI in a well-established transgenic mouse model of PD overexpressing human $\alpha$-synuclein (TNWT-61) under the murine Thy-1 promoter (59). This model is characterized by accumulation of $\alpha$-synuclein in almost all brain regions while there is no loss of neurons (60). DKI was measured in four different age groups. A young adult 3 and 6 months old mice were used to represent the early stage of PD like disease. At this age DKI demonstrated an increase in MK in striatum and thalamus and RK increase in thalamus in both time-points while in the SN MK was significantly increased only at 6 months old transgenic animals. Furthermore, tract-based spatial statistics analysis recorded alterations in WM only at 6 months (61). The second study used adult 9 months old mice which are at the zenith of the $\alpha$-synuclein pathology. In this report, MK demonstrated a significant increase in $\mathrm{SN}$, striatum, sensorimotor cortex and thalamus. AK showed differences only in the striatum and thalamus, while RK showed difference only in thalamus. In the same study tract-based spatial statistics showed an increase in MK bilaterally in the external capsule, AK in left-sided external capsule, RK bilaterally in the external capsule, corpus callosum, cingulum, dorsal hippocampal commissure. The last study focused on the late stage of the pathology present in 
very old mice at 14 months of age. Consistently with the previous findings MK demonstrated an increase in SN, striatum, sensorimotor cortex, thalamus and this time also hippocampus.

Considering all the above studies we can see certain pathological progression using the kurtosis metrics. Furthermore, unlike FA, kurtosis could show a remarkable result already in early stages of the PD-like pathology. Interestingly the MK increase in SN goes in line with the clinical findings which also found an increase in MK of SN $(49,53)$. Conversely, tract-based spatial statistics analysis found also increases in kurtosis metric in some WM regions which is not in line with clinical findings $(50,52)$. This discrepancy could be due to neurodegenerative process in PD which is not present in the transgenic animal model used in this study. Hence, $\alpha$-synuclein accumulation could be the pathological process underlying the increase of kurtosis values while loss of neurons may cause the opposite result.

Taken together, so far, there are no animal studies using DKI in another model than the transgenic TNWT-61 mice. The available reports found increases in kurtosis metrics at all-time points. Interestingly, the first regions showing enhanced MK already at 3 months of age were thalamus and striatum while in the SN the change become significant at 6 months. Overall, similarly as in the clinical trials, MK was the most sensitive readout and DKI seems to be more useful in detecting early PD brain pathology than DTI.

\section{Alzheimer's disease (AlzD)}

$\mathrm{AlzD}$ is a progressive neurodegenerative disorder affecting cognitive functions and it is a common cause for dementia. Hitherto there is no curative treatment of AlzD (62). However, it was suggested that at early stage of the neurodegenerative process an innovative neuroprotective treatment may slow down or halt the progression of AlzD (63). Therefore, early diagnosis of AlzD is of paramount importance and it could also provide a better understanding of the microstructural changes and monitor therapeutic efficacy of new drugs. Mild cognitive impairment (MCI) believed to be the transitional phase between normal and AlzD patients (64). MCI is associated with development and progression of brain atrophy, inititation of apoptosis (activation of glial cells) and reduction of the neuronal volume (myelin and axonal loss) leading to changes in water diffusivity $(65,66)$. Protein accumulation is one of the early hallmark of AlzD characterized by hyper-phosphorylation of tau proteins which start to aggregate leading to neurofibrillary tangles formation inside the nerve cell bodies (67, 68). This aggregation causes a collapse of neuronal connectivity and eventually cell death (69). Furthermore, the mutation of the genes encoding amyloid precursor protein and presenilin 1 and 2 alters the synthesis of amyloid- $\beta\left(\mathrm{A} \beta_{42}\right)$ the main component of senile plaques and cause 
several familial forms of AlzD. Recent studies indicate that glial cells (e.g. astrocytes, oligodendrocytes) play an important role in neuronal connectivity and neuronal death in AlzD. Therefore, an early neuroimaging biomarker which can detect the pathological protein accumulation may be a useful tool to provide a precise early diagnosis of AlzD.

\section{Clinical studies}

Currently the diagnosis of AlzD by neuroimaging is widely used especially structural MRI because it is able to measure the volume of the brain especially the volume of hippocampus. Therefore, developing new MRI methods for early stage of interest for the diagnosis of AlzD, Numerous DTI has shown remarkable results in brain ageing and dementia studies, due to its ability to evaluate the WM alteration associated with the disease progression. However, the meta-analysis identified diffusivity read-outs more sensitive than FA (65). The first DKI study in AlzD patients done by Falangola el. at. They divided the subjects into three groups: control, MCI and AlzD patients with no gender and age difference. All kurtosis metrics of AlzD patients and MCI are decreased in the anterior corona radiate compared with controls. RK and MK were decreased in temporal oval, segmental temporal and the genu of the corpus callosum, while RK was decreased in segmented prefrontal white matter of AlzD patients compared to controls. In comparison of MCI group with the controls, MK and RK were decreased in the prefrontal oval. These findings can provide evidence that significant changes in kurtosis are capable to differentiate the early changes as in MCI as well as in the late stage of AlzD compared to controls (70). However, a different study done by Wang, Guo et al. 2015, also divided the subjects into three groups control, MCI and AlzD patients with gender and age as the main difference between them. This study focused on hippocampus and a significant decrease of MK in MCI and AlzD patients was identified, also the lowest significant value was exhibited in AlzD patients. The authors explained these findings as results of the progression from normal brain tissue to MCI and then to AlzD. AlzD brain pathology is associated with the decrease in the structural complexity of the brain tissue and the lower tissue complexity the lower MK value (71). Furthermore, Gong et al. (2013), found kurtosis changes in four cerebral lobes. RK and MK were significantly decreased in WM of the parietal lobe. An increase of AK was found in the WM of the occipital lobe. No parameter was found to be significantly different between two groups in GM or WM of frontal and temporal lobes. This decrease in kurtosis in several cerebral lobes suggest that GM damage may expand from the temporal lobe to the Parieto-occipital association cortices as well as to frontal lobe, due to loss of neuronal cell bodies, synapse and dendrites (72). In conclusion, based on the previous reports, most of the 
studies were able to detect the difference between MCI, AlzD and controls in different brain regions. Interestingly, all these studies were also able to differentiate between AlzD and MCI using DKI, which shows that DKI may be a useful biomarker for early stage of AlzD. However, more investigation and data are needed to prove this hypothesis.

\section{Preclinical studies}

In case of preclinical AlzD research there is only one study available which evaluated the accumulation of amyloid- $\beta$ in a co-expressed KM670/671NL mutated amyloid precursor protein and L166P mutated presenilin 1 (APP/PS1) transgenic mice (73). This animal model is co-expressed by mutated presenilin 1 and mutated amyloid precursor protein which is believed to be a hallmark of AlzD. Using DKI and comparing it with the histopathological results, this study showed an increase in all kurtosis matrices in thalamus and cortex. This results corresponded to a histopathological evaluation of amyloid- $\beta$ accumulation which may increase the microstructural complexity and neuronal loss of the brain tissue $(74,75)$ which can explain the kurtosis increase in both regions. The limitation of this study is the lack of evidence of causal relationship between kurtosis and amyloid- $\beta$ accumulation. However, this report is a start for further investigation in animal models which could provide a better understanding the exact link between the AlzD-like pathology and kurtosis changes. Longitudinal study with DKI might prove if DKI is effective in evaluation of the pathology progression in this animal model.

\section{Huntington's disease (HD)}

Huntington's disease (HD) is a neurodegenerative genetic disorder and is one of several trinucleotides repeat disorders. Huntington protein is coded by a gene called huntingtin gene, a repeated part of this gene in called trinucleotide repeat. Normally it varies in length between individuals, but when this repetition reaches certain point it alters huntingtin protein to form a mutilated protein called mutilated huntingtin protein, these mutilated proteins leads to a pathological change that causes HD (76). A large set of data studied the specific function of mutilated huntingtin protein at microstructural level and its pathological effect that causes HD. To date, there is no curative treatment for HD. However, a reliable non-invasive biomarker would allow us to follow the progression of HD and give us a better chance to develop an effective therapy. As DKI proved the sensitivity toward the pathological progression of PD and $\mathrm{AlzD}$, it could be a useful biomarker to predict the development of HD as well. Unfortunately, only few preclinical studies where done using DKI with no clinical data up till now. 


\section{Preclinical studies}

There are two HD preclinical studies using tgHD transgenic rat model expresses a truncated huntingtin cDNA fragment that encodes 51 CAG repeats (77). This model is characterized by a pathological brain progression similar to HD together with motor abnormalities. The first study found an increase of RK in striatum and external capsule and suggested that this changes in the striatum may appear due to a high degree of diffusion complexity and restriction, while the changes in the external capsule may reflect fiber composition or cell permeability (78). The second study used newborn animals of both genders and DKI was assessed at postnatal days 15 and 30 in WM as well as GM. All kurtosis metrics were increased in the postnatal day 30 compared to day 15 in cortex, caudate putamen, corpus callosum and anterior commissure while in external capsule only MK and RK were increased. AK increased in caudate putamen in tgHD. These studies reported a novel finding in both aged and newborn transgenic rats of HD. These observations in diffusion characteristics provided us evidence that DKI is sensitive toward brain developmental abnormalities in distinct white and gray matter structures, associated with HD.

\section{Conclusion}

The importance of early diagnosis in neurodegeneration is of paramount importance for the future development of neuroprotective treatments. Furthermore, such approach helps in better understanding of the mechanisms which underlay the pathology. Based on the studies we reviewed, changes in DKI matrices reflects the structural complexity which may occur due to neuroinflammation such as immune activation and protein accumulation. Additionally, DKI showed a remarkable Effect in detecting the changes in GM over DTI in neurodegeneration diseases. This could bring the importance of DKI in as an early biomarker neurodegenerative diseases. Despite this, more data is needed to prove the validity of DKI as an early biomarker. 


\section{Acknowledgements}

This study was written at Masaryk University as part of the projects number MUNI/A/1063/2016 and MUNI/A/1132/2017 with the support of the Specific University Research Grant, as provided by the Ministry of Education, Youth and Sports of the Czech Republic.

\section{Conflict of interest statement}

All authors declare no conflict of interest. 


\section{References:}

1. Smith-Bindman R, Miglioretti DL, Johnson E, Lee C, Feigelson HS, Flynn M, et al. Use of diagnostic imaging studies and associated radiation exposure for patients enrolled in large integrated health care systems, 1996-2010. Jama. 2012;307(22):2400-9. Epub 2012/06/14.

2. Frank LR. Anisotropy in high angular resolution diffusion-weighted MRI. Magnetic resonance in medicine. 2001;45(6):935-9. Epub 2001/05/30.

3. Panigrahy A, Borzage M, Bluml S. Basic principles and concepts underlying recent advances in magnetic resonance imaging of the developing brain. Seminars in perinatology. 2010;34(1):3-19. Epub 2010/01/30.

4. Svolos P, Kousi E, Kapsalaki E, Theodorou K, Fezoulidis I, Kappas C, et al. The role of diffusion and perfusion weighted imaging in the differential diagnosis of cerebral tumors: a review and future perspectives. Cancer imaging : the official publication of the International Cancer Imaging Society. 2014;14:20. Epub 2015/01/23.

5. Johansen-Berg H, Behrens TE. Diffusion MRI: from quantitative measurement to in vivo neuroanatomy: Academic Press; 2013.

6. Gierek T, Paluch J, Pencak P, Kazmierczak B, Klimczak-Golab L. [Magnetic resonance tractography in neuroradiological diagnostic aspects]. Otolaryngologia polska $=$ The Polish otolaryngology. 2009;63(5):403-6. Epub 2010/02/23. Traktografia rezonansu magnetycznego $\mathrm{w}$ diagnostyce neuroradiologicznej.

7. Stejskal EO, Tanner JE. Spin diffusion measurements: spin echoes in the presence of a time-dependent field gradient. The journal of chemical physics. 1965;42(1):288-92.

8. Chilla GS, Tan $\mathrm{CH}, \mathrm{Xu} \mathrm{C}$, Poh CL. Diffusion weighted magnetic resonance imaging and its recent trend-a survey. Quantitative imaging in medicine and surgery. 2015;5(3):407-22. Epub 2015/06/02.

9. Ulug AM, Beauchamp N, Jr., Bryan RN, van Zijl PC. Absolute quantitation of diffusion constants in human stroke. Stroke. 1997;28(3):483-90. Epub 1997/03/01.

10. Fung SH, Roccatagliata L, Gonzalez RG, Schaefer PW. MR diffusion imaging in ischemic stroke. Neuroimaging clinics of North America. 2011;21(2):345-77, xi. Epub 2011/06/07.

11. Basser PJ. Inferring microstructural features and the physiological state of tissues from diffusion-weighted images. NMR in biomedicine. 1995;8(7-8):333-44. Epub 1995/11/01.

12. Basser P, Pierpaoli C. Recollections about our 1996 JMR paper on diffusion anisotropy. J Magn Reson. 2011;213(2):571-2. Epub 2011/12/14.

13. Einstein A. Über die von der molekularkinetischen Theorie der Wärme geforderte Bewegung von in ruhenden Flüssigkeiten suspendierten Teilchen. Annalen der Physik. 1905;322(8):549-60.

14. Assaf Y, Cohen Y. Assignment of the water slow-diffusing component in the central nervous system using q-space diffusion MRS: implications for fiber tract imaging. Magnetic resonance in medicine. 2000;43(2):191-9. Epub 2000/02/19.

15. Basser PJ. New histological and physiological stains derived from diffusion-tensor MR images. Annals of the New York Academy of Sciences. 1997;820:123-38. Epub 1997/05/30.

16. Westin CF, Maier SE, Mamata H, Nabavi A, Jolesz FA, Kikinis R. Processing and visualization for diffusion tensor MRI. Medical image analysis. 2002;6(2):93-108. Epub 2002/06/05.

17. Feldman HM, Yeatman JD, Lee ES, Barde LH, Gaman-Bean S. Diffusion tensor imaging: a review for pediatric researchers and clinicians. Journal of developmental and behavioral pediatrics: JDBP. 2010;31(4):346. 
18. Jensen JH, Helpern JA. MRI quantification of non-Gaussian water diffusion by kurtosis analysis. NMR in biomedicine. 2010;23(7):698-710. Epub 2010/07/16.

19. Tabesh A, Jensen JH, Ardekani BA, Helpern JA. Estimation of tensors and tensorderived measures in diffusional kurtosis imaging. Magnetic resonance in medicine. 2011;65(3):823-36. Epub 2011/02/22.

20. Viallon M, Cuvinciuc V, Delattre B, Merlini L, Barnaure-Nachbar I, Toso-Patel S, et al. State-of-the-art MRI techniques in neuroradiology: principles, pitfalls, and clinical applications. Neuroradiology. 2015;57(5):441-67. Epub 2015/04/11.

21. Jensen JH, Helpern JA, Ramani A, Lu H, Kaczynski K. Diffusional kurtosis imaging: the quantification of non-gaussian water diffusion by means of magnetic resonance imaging. Magnetic resonance in medicine. 2005;53(6):1432-40. Epub 2005/05/21.

22. Alexander AL, Hasan K, Kindlmann G, Parker DL, Tsuruda JS. A geometric analysis of diffusion tensor measurements of the human brain. Magnetic resonance in medicine. 2000;44(2):283-91. Epub 2000/08/05.

23. Steven AJ, Zhuo J, Melhem ER. Diffusion kurtosis imaging: an emerging technique for evaluating the microstructural environment of the brain. AJR American journal of roentgenology. 2014;202(1):W26-33. Epub 2013/12/29.

24. Meijer FJ, Bloem BR, Mahlknecht P, Seppi K, Goraj B. Update on diffusion MRI in Parkinson's disease and atypical parkinsonism. Journal of the neurological sciences. 2013;332(1-2):21-9. Epub 2013/07/23.

25. Wu EX, Cheung MM. MR diffusion kurtosis imaging for neural tissue characterization. NMR in biomedicine. 2010;23(7):836-48. Epub 2010/07/14.

26. Alexander AL, Lee JE, Lazar M, Field AS. Diffusion tensor imaging of the brain. Neurotherapeutics : the journal of the American Society for Experimental NeuroTherapeutics. 2007;4(3):316-29. Epub 2007/06/30.

27. Rovaris M, Filippi M. Diffusion tensor MRI in multiple sclerosis. Journal of neuroimaging : official journal of the American Society of Neuroimaging. 2007;17 Suppl 1:27S-30S. Epub 2007/04/12.

28. Rovaris M, Agosta F, Pagani E, Filippi M. Diffusion tensor MR imaging. Neuroimaging clinics of North America. 2009;19(1):37-43. Epub 2008/12/10.

29. Fellgiebel A, Wille P, Muller MJ, Winterer G, Scheurich A, Vucurevic G, et al. Ultrastructural hippocampal and white matter alterations in mild cognitive impairment: a diffusion tensor imaging study. Dementia and geriatric cognitive disorders. 2004;18(1):101-8. Epub 2004/04/17.

30. Kiuchi K, Morikawa M, Taoka T, Nagashima T, Yamauchi T, Makinodan M, et al. Abnormalities of the uncinate fasciculus and posterior cingulate fasciculus in mild cognitive impairment and early Alzheimer's disease: a diffusion tensor tractography study. Brain research. 2009;1287:184-91. Epub 2009/06/30.

31. Cochrane CJ, Ebmeier KP. Diffusion tensor imaging in parkinsonian syndromes: a systematic review and meta-analysis. Neurology. 2013;80(9):857-64. Epub 2013/02/27.

32. Chaudhary N, Pandey AS, Gemmete JJ, Hua Y, Huang Y, Gu Y, et al. Diffusion tensor imaging in hemorrhagic stroke. Experimental neurology. 2015;272:88-96. Epub 2015/05/28.

33. Dodd AB, Epstein K, Ling JM, Mayer AR. Diffusion tensor imaging findings in semiacute mild traumatic brain injury. Journal of neurotrauma. 2014;31(14):1235-48. Epub 2014/05/02.

34. Zhuo J, Xu S, Proctor JL, Mullins RJ, Simon JZ, Fiskum G, et al. Diffusion kurtosis as an in vivo imaging marker for reactive astrogliosis in traumatic brain injury. NeuroImage. 2012;59(1):467-77. Epub 2011/08/13.

35. Guglielmetti C, Veraart J, Roelant E, Mai Z, Daans J, Van Audekerke J, et al. Diffusion kurtosis imaging probes cortical alterations and white matter pathology following cuprizone 
induced demyelination and spontaneous remyelination. NeuroImage. 2016;125:363-77. Epub 2015/11/04.

36. Chaudhuri KR, Martinez-Martin P. Quantitation of non-motor symptoms in Parkinson's disease. European journal of neurology. 2008;15 Suppl 2:2-7. Epub 2008/08/22.

37. Pan-Montojo F, Funk RH. Implications of Parkinson's disease pathophysiology for the development of cell replacement strategies and drug discovery in neurodegenerative diseases. CNS \& neurological disorders drug targets. 2012;11(7):907-20. Epub 2012/11/08.

38. Braak H, Del Tredici K, Rub U, de Vos RA, Jansen Steur EN, Braak E. Staging of brain pathology related to sporadic Parkinson's disease. Neurobiology of aging. 2003;24(2):197-211. Epub 2002/12/25.

39. Desplats P, Lee HJ, Bae EJ, Patrick C, Rockenstein E, Crews L, et al. Inclusion formation and neuronal cell death through neuron-to-neuron transmission of alpha-synuclein. Proceedings of the National Academy of Sciences of the United States of America. 2009;106(31):13010-5. Epub 2009/08/05.

40. Rizek P, Kumar N, Jog MS. An update on the diagnosis and treatment of Parkinson disease. CMAJ : Canadian Medical Association journal = journal de l'Association medicale canadienne. 2016;188(16):1157-65. Epub 2016/11/02.

41. Prakash KG, Bannur BM, Chavan MD, Saniya K, Sailesh KS, Rajagopalan A. Neuroanatomical changes in Parkinson's disease in relation to cognition: An update. Journal of advanced pharmaceutical technology \& research. 2016;7(4):123-6. Epub 2016/11/12.

42. Angot E, Steiner JA, Lema Tome CM, Ekstrom P, Mattsson B, Bjorklund A, et al. Alpha-synuclein cell-to-cell transfer and seeding in grafted dopaminergic neurons in vivo. PloS one. 2012;7(6):e39465. Epub 2012/06/28.

43. Rascol O. Drugs and drug delivery in PD: optimizing control of symptoms with pramipexole prolonged-release. European journal of neurology. 2011;18 Suppl 1:3-10. Epub 2011/02/03.

44. Stocchi F, Olanow CW. Obstacles to the development of a neuroprotective therapy for Parkinson's disease. Movement disorders : official journal of the Movement Disorder Society. 2013;28(1):3-7. Epub 2013/02/08.

45. Vaillancourt DE, Spraker MB, Prodoehl J, Abraham I, Corcos DM, Zhou XJ, et al. High-resolution diffusion tensor imaging in the substantia nigra of de novo Parkinson disease. Neurology. 2009;72(16):1378-84. Epub 2009/01/09.

46. Lang AE, Mikulis D. A new sensitive imaging biomarker for Parkinson disease? Neurology. 2009;72(16):1374-5. Epub 2009/01/09.

47. Rolheiser TM, Fulton HG, Good KP, Fisk JD, McKelvey JR, Scherfler C, et al. Diffusion tensor imaging and olfactory identification testing in early-stage Parkinson's disease. Journal of neurology. 2011;258(7):1254-60. Epub 2011/02/03.

48. Ibarretxe-Bilbao N, Junque C, Marti MJ, Valldeoriola F, Vendrell P, Bargallo N, et al. Olfactory impairment in Parkinson's disease and white matter abnormalities in central olfactory areas: A voxel-based diffusion tensor imaging study. Movement disorders : official journal of the Movement Disorder Society. 2010;25(12):1888-94. Epub 2010/07/30.

49. Wang JJ, Lin WY, Lu CS, Weng YH, Ng SH, Wang CH, et al. Parkinson disease: diagnostic utility of diffusion kurtosis imaging. Radiology. 2011;261(1):210-7. Epub 2011/07/21.

50. Kamagata K, Tomiyama H, Motoi Y, Kano M, Abe O, Ito K, et al. Diffusional kurtosis imaging of cingulate fibers in Parkinson disease: comparison with conventional diffusion tensor imaging. Magnetic resonance imaging. 2013;31(9):1501-6. Epub 2013/07/31.

51. Smith SM, Jenkinson M, Johansen-Berg H, Rueckert D, Nichols TE, Mackay CE, et al. Tract-based spatial statistics: voxelwise analysis of multi-subject diffusion data. NeuroImage. 2006;31(4):1487-505. Epub 2006/04/21. 
52. Kamagata K, Tomiyama H, Hatano T, Motoi Y, Abe O, Shimoji K, et al. A preliminary diffusional kurtosis imaging study of Parkinson disease: comparison with conventional diffusion tensor imaging. Neuroradiology. 2014;56(3):251-8. Epub 2014/01/29.

53. Zhang G, Zhang Y, Zhang C, Wang Y, Ma G, Nie K, et al. Diffusion Kurtosis Imaging of Substantia Nigra Is a Sensitive Method for Early Diagnosis and Disease Evaluation in Parkinson's Disease. Parkinson's disease. 2015;2015:207624. Epub 2016/01/16.

54. Chan LL, Rumpel H, Yap K, Lee E, Loo HV, Ho GL, et al. Case control study of diffusion tensor imaging in Parkinson's disease. Journal of neurology, neurosurgery, and psychiatry. 2007;78(12):1383-6. Epub 2007/07/07.

55. Zhan W, Kang GA, Glass GA, Zhang Y, Shirley C, Millin R, et al. Regional alterations of brain microstructure in Parkinson's disease using diffusion tensor imaging. Movement disorders : official journal of the Movement Disorder Society. 2012;27(1):90-7. Epub 2011/08/19.

56. Van Camp N, Blockx I, Verhoye M, Casteels C, Coun F, Leemans A, et al. Diffusion tensor imaging in a rat model of Parkinson's disease after lesioning of the nigrostriatal tract. NMR in biomedicine. 2009;22(7):697-706. Epub 2009/04/21.

57. Boska MD, Hasan KM, Kibuule D, Banerjee R, McIntyre E, Nelson JA, et al. Quantitative diffusion tensor imaging detects dopaminergic neuronal degeneration in a murine model of Parkinson's disease. Neurobiology of disease. 2007;26(3):590-6. Epub 2007/04/13.

58. Hikishima K, Ando K, Yano R, Kawai K, Komaki Y, Inoue T, et al. Parkinson Disease: Diffusion MR Imaging to Detect Nigrostriatal Pathway Loss in a Marmoset Model Treated with 1-Methyl-4-phenyl-1,2,3,6-tetrahydropyridine. Radiology. 2015;275(2):430-7. Epub 2015/01/21.

59. Masliah E, Rockenstein E, Veinbergs I, Mallory M, Hashimoto M, Takeda A, et al. Dopaminergic loss and inclusion body formation in alpha-synuclein mice: implications for neurodegenerative disorders. Science. 2000;287(5456):1265-9. Epub 2000/02/26.

60. Delenclos M, Carrascal L, Jensen K, Romero-Ramos M. Immunolocalization of human alpha-synuclein in the Thy1-aSyn ("Line 61") transgenic mouse line. Neuroscience. 2014;277:647-64. Epub 2014/08/06.

61. Khairnar A, Ruda-Kucerova J, Szabo N, Drazanova E, Arab A, Hutter-Paier B, et al. Early and progressive microstructural brain changes in mice overexpressing human alphaSynuclein detected by diffusion kurtosis imaging. Brain, behavior, and immunity. 2016. Epub 2016/12/08.

62. Braak H, Braak E. Development of Alzheimer-related neurofibrillary changes in the neocortex inversely recapitulates cortical myelogenesis. Acta neuropathologica. 1996;92(2):197-201. Epub 1996/08/01.

63. Godyn J, Jonczyk J, Panek D, Malawska B. Therapeutic strategies for Alzheimer's disease in clinical trials. Pharmacological reports : PR. 2016;68(1):127-38. Epub 2016/01/02.

64. Batum K, Cinar N, Sahin S, Cakmak MA, Karsidag S. The connection between MCI and Alzheimer disease: neurocognitive clues. Turkish journal of medical sciences. 2015;45(5):1137-40. Epub 2016/01/08.

65. Clerx L, Visser PJ, Verhey F, Aalten P. New MRI markers for Alzheimer's disease: a meta-analysis of diffusion tensor imaging and a comparison with medial temporal lobe measurements. Journal of Alzheimer's disease : JAD. 2012;29(2):405-29. Epub 2012/02/15.

66. Falangola MF, Jensen JH, Babb JS, Hu C, Castellanos FX, Di Martino A, et al. Agerelated non-Gaussian diffusion patterns in the prefrontal brain. Journal of magnetic resonance imaging : JMRI. 2008;28(6):1345-50. Epub 2008/11/26.

67. Selkoe DJ. Alzheimer's disease: genes, proteins, and therapy. Physiological reviews. 2001;81(2):741-66. Epub 2001/03/29. 
68. Selkoe DJ, Schenk D. Alzheimer's disease: molecular understanding predicts amyloidbased therapeutics. Annual review of pharmacology and toxicology. 2003;43:545-84. Epub 2002/11/05.

69. Gotz J, Schild A, Hoerndli F, Pennanen L. Amyloid-induced neurofibrillary tangle formation in Alzheimer's disease: insight from transgenic mouse and tissue-culture models. International journal of developmental neuroscience : the official journal of the International Society for Developmental Neuroscience. 2004;22(7):453-65. Epub 2004/10/07.

70. Falangola MF, Jensen JH, Tabesh A, Hu C, Deardorff RL, Babb JS, et al. Non-Gaussian diffusion MRI assessment of brain microstructure in mild cognitive impairment and Alzheimer's disease. Magnetic resonance imaging. 2013;31(6):840-6. Epub 2013/04/23.

71. Wang D, Guo ZH, Liu XH, Li YH, Wang H. Examination of hippocampal differences between Alzheimer disease, amnestic mild cognitive impairment and normal aging: diffusion kurtosis. Current Alzheimer research. 2015;12(1):80-7. Epub 2014/12/20.

72. Gong NJ, Wong CS, Chan CC, Leung LM, Chu YC. Correlations between microstructural alterations and severity of cognitive deficiency in Alzheimer's disease and mild cognitive impairment: a diffusional kurtosis imaging study. Magnetic resonance imaging. 2013;31(5):688-94. Epub 2013/01/26.

73. Vanhoutte G, Pereson S, Delgado YPR, Guns PJ, Asselbergh B, Veraart J, et al. Diffusion kurtosis imaging to detect amyloidosis in an APP/PS1 mouse model for Alzheimer's disease. Magnetic resonance in medicine. 2013;69(4):1115-21. Epub 2013/03/16.

74. Matsuoka Y, Picciano M, Malester B, LaFrancois J, Zehr C, Daeschner JM, et al. Inflammatory responses to amyloidosis in a transgenic mouse model of Alzheimer's disease. The American journal of pathology. 2001;158(4):1345-54. Epub 2001/04/06.

75. Wengenack TM, Whelan S, Curran GL, Duff KE, Poduslo JF. Quantitative histological analysis of amyloid deposition in Alzheimer's double transgenic mouse brain. Neuroscience. 2000;101(4):939-44. Epub 2000/12/13.

76. Walker FO. Huntington's disease. Lancet. 2007;369(9557):218-28. Epub 2007/01/24. 77. von Horsten S, Schmitt I, Nguyen HP, Holzmann C, Schmidt T, Walther T, et al. Transgenic rat model of Huntington's disease. Human molecular genetics. 2003;12(6):617-24. Epub 2003/03/07.

78. Blockx I, Verhoye M, Van Audekerke J, Bergwerf I, Kane JX, Delgado YPR, et al. Identification and characterization of Huntington related pathology: an in vivo DKI imaging study. NeuroImage. 2012;63(2):653-62. Epub 2012/06/30. 


\section{Figure legends}

\section{Figure 1}

Illustration of diffusion displacement probability distribution with different degrees of kurtosis, where $\mathrm{K}=0$ is a Gaussian and $\mathrm{K}>0$ is non-Gaussian water distribution. Mathematically $\mathrm{K}<$ 0 is possible, but multicompartment diffusion models and empirical evidence indicate that kurtosis is always nonnegative (19).

\section{Figure 2}

Scheme of diffusion MR using spin echo sequence and illustration of the physical quantities of the $b$-value. Received signal from stationery molecules are unaffected, while free water molecules move into different locations, diphase and consequently the signal is lost.

\section{Figure 3}

Schematic representation of diffusion tensor ellipsoid $\left(\mathrm{e}_{1}, \mathrm{e}_{2}, \mathrm{e}_{3}\right.$ are eigenvectors; $\lambda_{1}, \lambda_{2}, \lambda_{3}$ are eigenvalues) and distribution of kurtosis.

\section{Figure 4}

Illustration of MR signal loss curve as a function of b-value for Gaussian (DTI) and nonGaussian (DKI) models of water diffusion in brain. 